\title{
miR-155-5p increases the sensitivity of liver cancer cells to adriamycin by regulating ATG5-mediated autophagy
}

\author{
Qiu YU, Xiao-Ping XU, Xin-Min YIN, Xiang-Qun PENG*
}

Hepatobiliary Department I, Hunan Provincial People's Hospital, The First Affiliated Hospital of Hunan Normal University, The College of Clinical Medicine of Hunan Normal University, Changsha, China

${ }^{*}$ Correspondence: 42942514@qq.com

Received January 6, 2020 / Accepted June 17, 2020

\begin{abstract}
Liver cancer is the sixth most prevalent cancer worldwide and the third leading cause of cancer-related deaths. Adriamycin (ADR) resistance, which often leads to the progression of malignant tumors, is a major treatment obstacle for liver cancer. It has been confirmed that miR-155-5p could reverse drug resistance in human breast cancer. However, the biological function of miR-155-5p in ADR-resistant liver carcinoma (HepG2/ADR) cells remains unclear. miR-155-5p and ATG5 expression was determined by RT-qPCR and western blot. In addition, MTT, flow cytometry, immunofluorescence staining, and western blotting were performed to evaluate the proliferation, apoptosis, and autophagy of liver cancer cells. Finally, the effect of miR-155-5p on the expression of autophagy-related 5 (ATG5) was analyzed by luciferase activity assay, western blot, and RT-qPCR. Our results showed that miR-155-5p was downregulated in HepG2/ADR cells. Increasing the expression of miR-155-5p enhanced the sensitivity of liver carcinoma cells to ADR and promoted apoptosis through inhibition of autophagy in vitro. In addition, the binding site between miR-155-5p and ATG5 was identified, and miR-155-5p could directly regulate ATG5. Finally, ATG5 partially rescued the effect of miR-155-5p on autophagy and the apoptosis of HepG2/ ADR cells. In conclusion, our findings showed that miR-155-5p could reverse ADR resistance in liver cancer by targeting ATG5, which may function as a potential target for liver cancer treatment.
\end{abstract}

Key words: resistance, autophagy, miR-155-5p, liver cancer

Liver cancer is considered a major incurable disease [1]. Liver cancer remains one of the most difficult cancers to treat, its incidence is rapidly increasing, and its survival rates vary by country, especially in China. Moreover, less than $20 \%$ of patients with this malignant tumor are still alive more than half a year after diagnosis [1-4]. For many years, efforts towards the treatment of liver cancer were focused on the liver cancer cells themselves. Currently, the major treatment strategy is chemotherapy. However, the prognostic effect of liver cancer treatment is still limited. The major cause for the poor prognosis of liver cancer is the chemoresistance of carcinoma cells, especially to adriamycin (ADR), which occurs in approximately $50 \%$ of patients with advanced liver carcinoma undergoing chemotherapy [5]. Therefore, studying the specific molecular mechanisms of ADR resistance in liver cancer to find new drug targets and improve the survival rate of patients with liver cancer is of great importance.

MicroRNAs (miRNAs) are a group of endogenous non-coding RNAs (ncRNAs) that function through mRNA regulation or downregulation of translation by associating with the 3'-untranslated region (3'-UTR) of their target genes [6]. MiRNAs play key roles in multiple biological processes, including proliferation and apoptosis [7, 8]. Additionally, miRNAs can function as both promoters and suppressors of malignant tumors. Furthermore, their potential to regulate numerous target genes makes miRNAs a promising tool in the fight against malignancies. For instance, recent reports have indicated that miRNAs can regulate the tumorigenesis, progression, and pathogenesis of liver cancer [9-12]. In particular, $\mathrm{Ma}$ et al. found that miRNAs could relieve resistance to TRAIL-induced cell growth inhibition in liver cancer [13]. Since then, a number of miRNAs have been confirmed to modulate chemoresistance in liver cancer cells $[6,14]$. Thus, miRNAs involved in chemoresistance in liver cancer can provide new targets for the treatment of liver cancer. Moreover, miR-155-5p has been found to regulate the proliferation, migration, and invasion of cancer cells [15-17]. Zhang et al. revealed that miR-155-5p could rescue paclitaxel resistance through negative regulation of MYD88 in human breast cancer [18]. However, the effect of miR-155-5p in ADR-resistant liver cancer remains unclear. 
Autophagy is known to participate in the biological processes of many diseases [19]. There are approximately 30 autophagy-related genes (Atgs) with multiple mammalian genetic homologs that have been confirmed [20]. Atgs are necessary for adapting to microenvironmental stresses, especially heat stress and the accumulation of reactive oxygen species (ROS) [20]. Autophagy could be involved in a series of processes, including lysosomal fusion and initiation of phagophore assembly [21]. Recent studies have suggested that inhibition of autophagy can sensitize cancer cells to chemoresistance $[22,23]$. Sui et al. found that ATG5 could be involved in chemoresistance in malignant tumors [24]. Therefore, we hypothesized that autophagy might act as a key regulator in ADR-resistant liver cancer.

Overall, we sought to detect the effect of miR-155-5p on ADR-resistant liver cancer and its underlying mechanism. Our study first found that miR-155-5p could reverse ADR resistance in liver cancer through the inhibition of autophagy by directly targeting ATG5, which provided a new strategy for the treatment of liver cancer.

\section{Material and methods}

Cell culture. HepG2 and HepG2/ADR cell lines were provided by the Shanghai Institute of Biochemistry and Cell Biology, CAS (Shanghai, China). Cells were cultured in RPMI-1640 (Thermo Fischer Scientific, USA) with 10\% fetal bovine serum (FBS, Thermo Fischer Scientific), 1\% penicillin (Invitrogen) and streptomycin (Thermo Fischer Scientific) at $37^{\circ} \mathrm{C}$ and $5 \% \mathrm{CO}_{2}$. ADR-resistant HepG2/ADR cells were maintained via supplementation with $1 \mu \mathrm{g} / \mathrm{ml}$ ADR. In addition, HepG2/ADR cells were cultured in drugfree medium for two weeks before the following experiments.

MTT assay. To detect drug resistance, HepG2 and HepG2/ADR cells were treated with different concentrations of $\operatorname{ADR}(0,0.1,0.5,1,5,10$, and $50 \mu \mathrm{M})$ for $48 \mathrm{~h}$. An MTT assay was then carried out to quantify cell viability. First, 10 $\mu \mathrm{l}$ of MTT solution and $100 \mu \mathrm{l}$ of medium were mixed and added per well to the cells for $4 \mathrm{~h}$. Then, the solution was discarded and $150 \mu \mathrm{l}$ of DMSO was added to each well. After the plates were slightly shaken for $10 \mathrm{~min}$, the absorbance at $490 \mathrm{~nm}$ was determined with a spectrophotometer. The half-maximal inhibitory concentration (IC50) values were calculated by nonlinear regression analysis using GraphPad Prism version 5.0 (GraphPad Software, Inc.) software as previously described [25].
Cell transfection. miR-155-5p mimic/inhibitor and negative control duplex with a random non-targeting sequence (names NC mimic and NC inhibitor) were all synthesized by GenePharma (Shanghai, China). The ATG5 or BCL2-expressing pcDNA3.1 plasmid was purchased from Origene (Beijing, China). The cells were transfected with a mixture containing Lipofectamine 2000 reagent (Invitrogen, USA) and miR-155-5p/NC mimic or miR-155-5p/NC inhibitor. Subsequently, the HepG2/ADR cells were treated with ADR or left untreated. RNA extraction and western blot experiments were conducted at $48 \mathrm{~h}$.

Reverse transcription-quantitative PCR (RT-qPCR). Total RNA from HepG2 or HepG2/ADR cells was isolated by using TRIzol (Invitrogen, USA) as described by the manufacturer's instructions. Then, Prime Script RT Master Mix (TaKaRa, Japan) was used to reverse transcribe RNA into cDNA. Subsequently, real-time PCR was performed according to the direction of the SYBR ${ }^{\mathrm{TM}}$ Green master mix (TaKaRa, Japan). The relative expression levels were quantified using the $2^{-\triangle \Delta C t}$ method. GAPDH or U6 was used as an internal control. Primer sequences are listed in Table 1.

Immunofluorescence staining assay. HepG2 or HepG2/ ADR cells were cultured on glass coverslips until $80 \%$ confluence and then fixed with $4 \%$ paraformaldehyde for $30 \mathrm{~min}$. Next, cells were blocked with $10 \%$ goat serum for $30 \mathrm{~min}$ at room temperature and then incubated with the primary antibody anti-LC3II (ab232940, Abcam; 1:500) at $4{ }^{\circ} \mathrm{C}$ overnight, followed by the incubation with the secondary antibody goat anti-rabbit IgG (Abcam; $1: 2000)$ at $37^{\circ} \mathrm{C}$ for $1 \mathrm{~h}$. Then, the nuclei were stained with DAPI (Beyotime, Shanghai, China) for $5 \mathrm{~min}$. Finally, the cells were imaged by a fluorescence microscope (Nikon, Japan).

Cell apoptosis assay. An annexin V-FITC apoptosis detection kit (Beyotime, Shanghai, China) was used to test cell apoptosis according to the protocol. Cells were doublestained with annexin V-FITC and propidium iodide (PI), followed by incubation in the dark at room temperature for $20 \mathrm{~min}$. Apoptotic cells were analyzed using a flow cytometer. Data were assessed using BD FACSDiva Software version 7.0 (Becton-Dickinson, USA).

Western blotting assay. RIPA buffer was used to isolate total protein from cell lysates. In addition, proteins were further quantified by a BCA protein assay kit (Beyotime). Proteins were separated by using a 10\% SDS-PAGE gel, and proteins were then transferred to PVDF membranes (Millipore, USA). The PVDF membranes were blocked with 5\%

Table 1. Primer sequences for quantitative real-time PCR.

\begin{tabular}{lll}
\hline Gene & Forward sequence & Reverse sequence \\
\hline hsa-miR-155-5p & 5'-GAGGGTTAATGCTAAT CGTGATAGG-3' & 5'-GCACAGAATCA ACACGACTCACTAT-3' \\
ATG5 & 5'-AAGACCTTCTGCACTGTCCA-3' & 5'-GAGTTTCCGATTGATGGCCC-3' \\
U6 & 5'-CTCGCTTCGGCAGCACA-3' & 5'-AACGCTTCACGAATTTGCGT-3' \\
GAPDH & 5'-CCAGGTGGTCTCCTCTGA-3' & 5'-GCTGTAGCCAAATCGTTGT-3' \\
\hline
\end{tabular}


skim milk in TBST at room temperature for $1 \mathrm{~h}$. After that, the PVDF membranes were incubated with the following primary antibodies (Abcam): anti-LC3B (ab51520; 1:3000, with specificity to LC3I and LC3II), anti-ATG5 (ab108327; 1:2000), anti-Beclin-1 (ab217179; 1:1000), anti-Bax (ab32503; 1:1000), anti-BCL2 (ab59348; 1:500), anti-cleaved caspase-3 (ab2302; 1:1000), anti-cleaved caspase-9 (ab2324; 1:1000), and anti-GAPDH (ab9485; 1:2500) overnight at $4{ }^{\circ} \mathrm{C}$. After that, the PVDF membranes were incubated with the secondary anti-rabbit antibody for $1 \mathrm{~h}$ at room temperature. Finally, the bands were analyzed by an ECL detection kit (Pierce Biotechnology, USA). The relative protein expression was normalized to GAPDH expression.

Dual-luciferase reporter assay. The dual-luciferase reporter assay was performed as previously described, with a slight modification [26]. The predicted miR-155-5p binding site on the ATG5 3'-UTR sequence or mutant sequence was cloned into the psiCHECK-2 luciferase vector (Promega, USA) to generate the ATG5 wild-type (wt) luciferase reporter. Subsequently, HepG2/ADR cells were seeded and cultured in cell plates and were co-transfected with the wildtype ATG5 3'-UTR vector or mutant ATG5 3'-UTR vector and miR-155-5p/NC mimic using Lipofectamine 2000 (Invitrogen). Then, the luciferase activity was detected by the Dual-Luciferase Reporter Assay System (Promega, USA).

Statistical analysis. GraphPad Prism 5.0 was applied for statistical analysis. Data are represented as the mean \pm standard deviation (SD). All experiments were performed in triplicate. The comparison between the two groups was analyzed by Student's t-test. The comparisons among multiple groups were made with one-way analysis of variance (ANOVA) followed by Tukey's test. A p-value $<0.05$ was considered a statistically significant difference.

\section{Results}

Hepatocellular carcinoma cell line HepG2/ADR exhibits chemotherapeutic resistance and enhanced autophagy activity. In our study, we found that the IC50 of ADR was obviously higher in HepG2/ADR cells than in HepG2 cells $(20.51 \mu \mathrm{M}$ vs. $0.89 \mu \mathrm{M}$, Figure $1 \mathrm{~A})$. This result suggested that HepG2/ADR cells were highly resistant to ADR. Next, to further explore the function of autophagy in chemoresistance in liver cancer, immunofluorescence staining assay was used. As indicated in Figure 1B, LC3 was notably upregulated in HepG2/ADR cells compared with parental HepG2 cells. Moreover, the expression of LC3II was notably enhanced in HepG2/ADR cells (Figure 1C). In addition, miR-155-5p expression was greatly downregulated in HepG2/ADR cells compared with HepG2 cells (Figure 1D). All these data demonstrated that the HepG2/ADR cell line had better autophagy activity than parental HepG2 cells.

Overexpression of miR-155-5p sensitized HepG2/ADR cells to ADR by inhibiting autophagy. For the purpose of verifying the regulatory effect of miR-155-5p on the resistance of HepG2/ADR cells to ADR, overexpression of miR-155-5p was conducted via transfection, and apoptosis and autophagy were determined. As shown in Figure 2A, the upregulation of miR-155-5p notably enhanced the expression of miR-155-5p compared with the NC mimic, which indicated
A

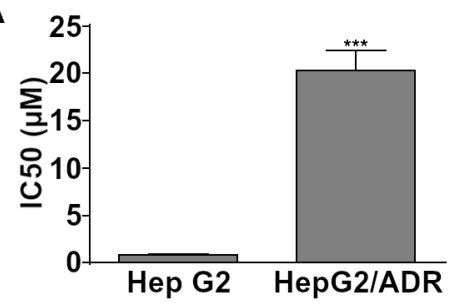

C

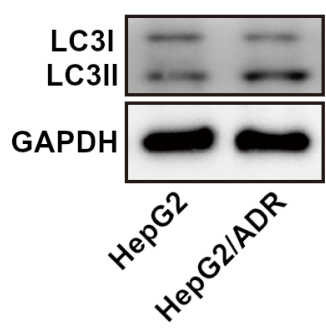

B

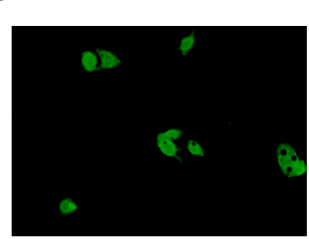

Hep G2

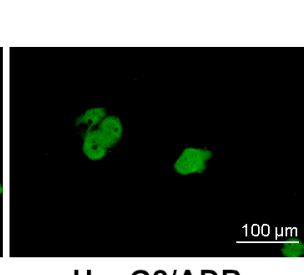

HepG2/ADR
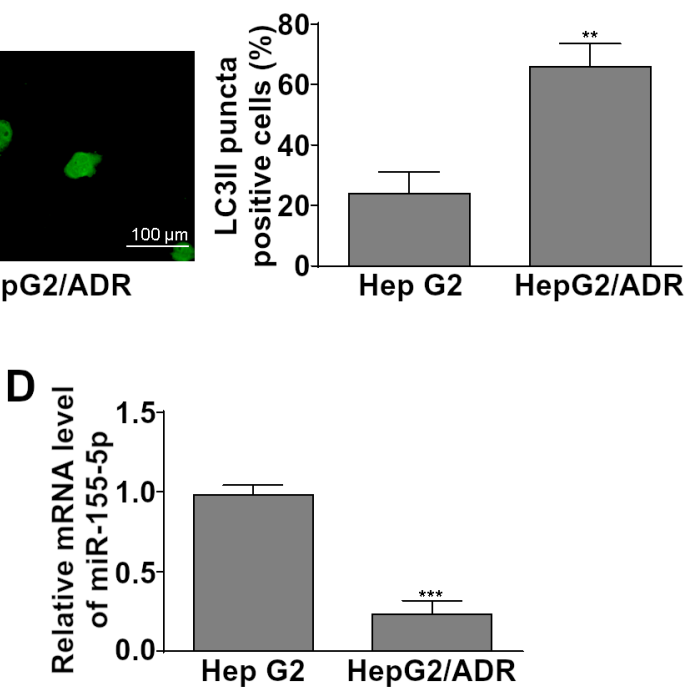

Figure 1. Hepatocellular carcinoma cell line HepG2/ADR exhibits chemotherapeutic resistance and enhanced autophagy activity. A) HepG2 and HepG2/ADR cells were treated with 0-50 MM ADR for $48 \mathrm{~h}$. Then, the IC50 value in cells was determined by MTT assay. B) After incubation for $48 \mathrm{~h}$, the expression of LC3 in HepG2 or HepG2/ADR cells was observed by immunofluorescence staining. C) The expression of LC3I and LC3II in HepG2/ ADR cells was detected by western blot. GAPDH was used as an internal control. D) miR-155-5p expression in HepG2 or HepG2/ADR cells was determined by RT-qPCR. U6 was used as an internal control. ${ }^{*} \mathrm{p}<0.05 ;{ }^{* *} \mathrm{p}<0.01$ 
A

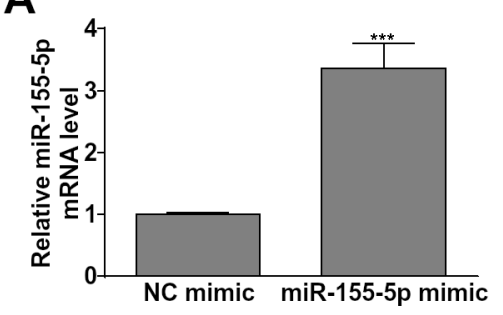

C

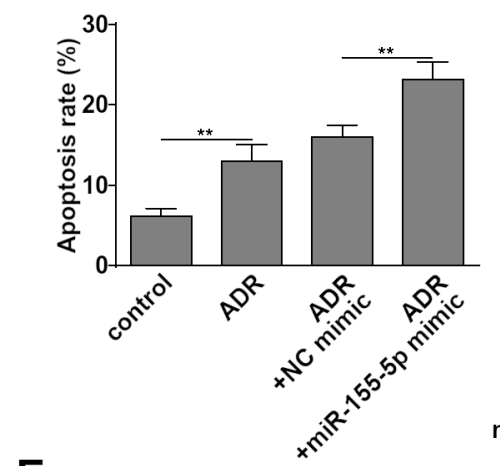

E

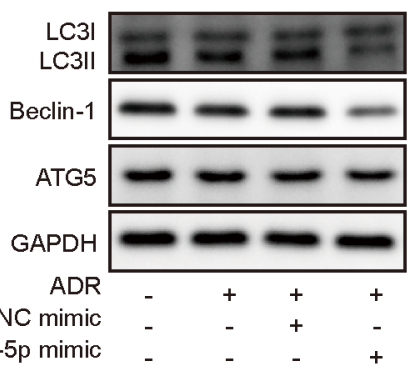

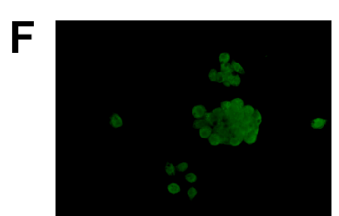

control

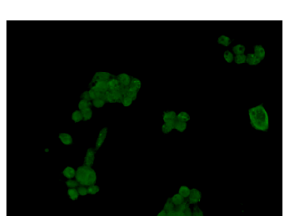

ADR
B

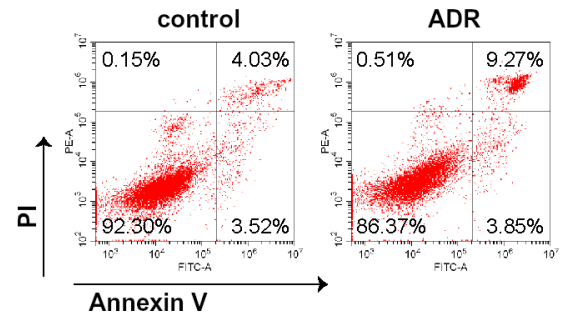

D

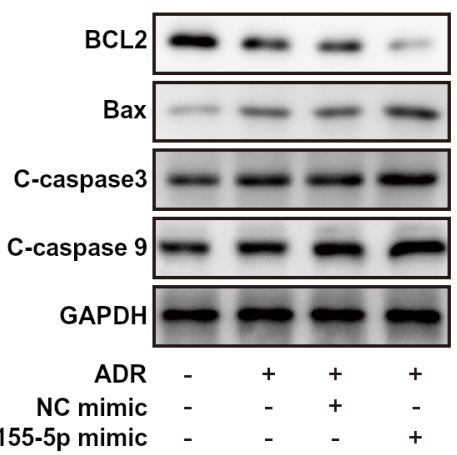

$\frac{1}{0}$

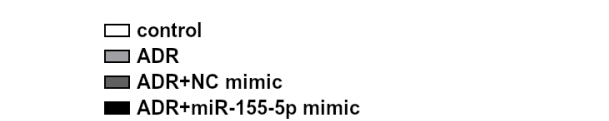

DRR+ mimic $\quad A D R+m i R-155-5 p$ mimic

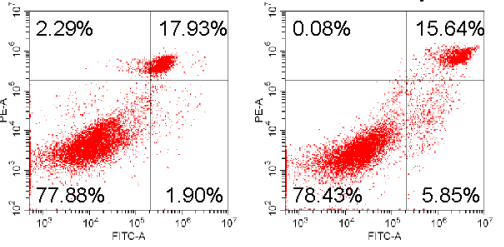

- ADR+miR-155-5p mimic

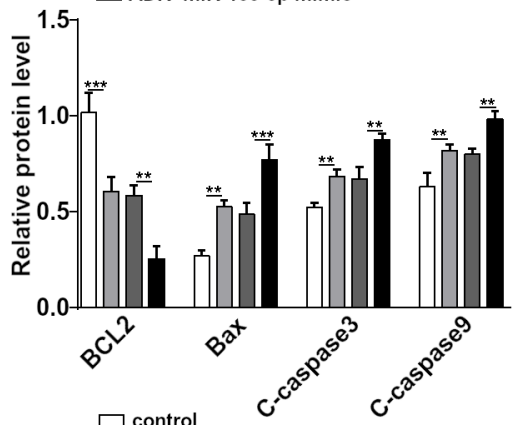

$\square \mathrm{ADR}$

$\square$ ADR+NC mimic

ADR+miR-155-5p mimic
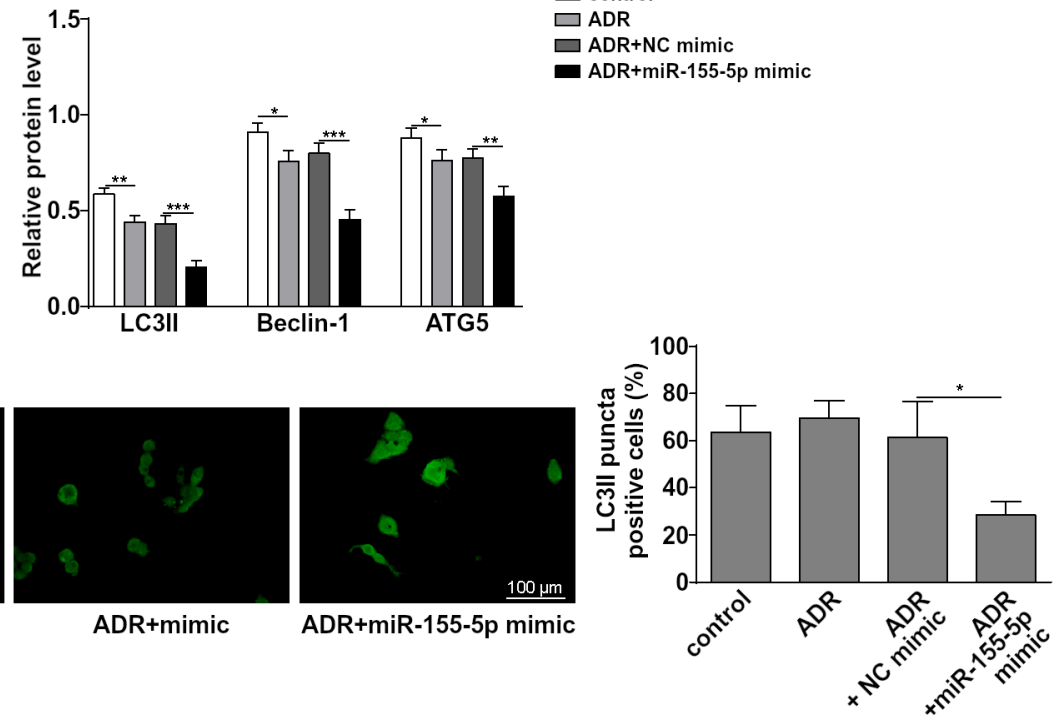

Figure 2. Overexpression of miR-155-5p sensitized HepG2/ADR cells to ADR by inhibiting autophagy. A) After incubation for 48 h, miR-155-5p expression in HepG2/ADR cells was measured by RT-qPCR. B) Apoptotic cells were quantified by FACS. C) Quantitative analysis of (B). D) The expression of cleaved caspase-3, cleaved caspase-9, Bax, and BCL2 was detected by western blot. E) The expression of LC3 was investigated by immunofluorescence staining. F) The expression of LC3II, LC3I, Beclin-1, and ATG5 was detected by western blot. ${ }^{*} p<0.05 ;{ }^{* *} p<0.01 ;{ }^{* * *} p<0.001$

that miR-155-5p was stably transfected into HepG2/ADR cells. Next, flow cytometry was performed to investigate the effect of miR-155-5p on the apoptosis of HepG2/ADR cells. As demonstrated in Figure 2B, ADR alone had a limited effect on the apoptosis of HepG2/ADR cells, while the miR-155-5p mimic notably promoted apoptosis in HepG2/ADR cells. Moreover, the expressions of apoptosis-related proteins were assessed by western blot. As illustrated in Figure 2C, the expression of BCL2 was significantly reduced, and Bax, cleaved caspase-3, and cleaved caspase-9 in HepG2/ADR cells were not notably activated by ADR, while the miR-155-5p mimic significantly reduced the levels of BCL2 but enhanced those of Bax, cleaved caspase-3, and cleaved caspase-9. Immunofluorescence assays showed that ADR alone did not significantly affect the expression of LC3, while overexpression of miR-155-5p notably enhanced the inhibitory effect of ADR on the expression of LC3 (Figure 2D). Finally, western blotting was performed to measure autophagy-related 
protein expression in HepG2/ADR cells. As indicated in Figure $2 \mathrm{E}$, the expression of autophagy-related proteins was slightly decreased in the presence of ADR, which was further inhibited by the miR-155-5p mimic. Collectively, these results revealed that overexpression of miR-155-5p sensitized HepG2 cells to ADR treatment by inhibiting autophagy.

ATG5 and Bcl-2 are direct binding targets of miR-155-5p. Next, the StarBase software was used to predict the downstream targets of miR-155-5p. We found that the 3'-UTRs of ATG5 and BCL2 bound to miR-155-5p (Figure 3A, Figure S1A). To verify the binding of miR-155-5p and ATG5/ BCL2, a dual-luciferase reporter assay was performed. The data suggested that luciferase activity was clearly decreased after co-transfection with the miR-155-5p mimic and wildtype ATG5/BCL2 vector but was not altered after co-transfection with the miR-155-5p mimic and mutant ATG5/BCL2 vector (Figure 3B, Figure S1B). Moreover, RT-qPCR analysis showed that ATG5/BCL2 was negatively regulated by the miR-155-5p mimic (Figures 3C and S1C), which was further confirmed by western blot (Figure 3D, Figure S1D). All these data suggest that miR-155-5p binds to ATG5 and negatively regulates its expression.

miR-155-5p sensitized HepG2/ADR cells to ADR by targeting ATG5/BCL2. Next, we conducted an experiment to validate the biological function of ATG5/BCL2 in miR-155-5p-mediated autophagy. As indicated in Figure 4A and Figures S2A and S2B, the expression of ATG5/BCL2 in HepG2/ADR cells was significantly inhibited in the presence of the miR-155-5p mimic, which was partially rescued by ATG5/BCL2 overexpression. Furthermore, the results indicated that the miR-155-5p mimic significantly induced apoptosis in HepG2/ADR cells, while ATG5 overexpression partially reversed the apoptotic effect of the miR-155-5p mimic (Figure 4B, Figure S2C). Indeed, as shown in Figure 4C, miR-155-5p overexpression significantly reduced the relative expression of BCL2 but enhanced the relative expression of pro-apoptotic proteins (Bax, cleaved caspase-3, and cleaved caspase-9). However, ATG5 overexpression partially reversed the effects on the expression levels. Immunofluorescence assays suggested that LC3 expression was notably downregulated in HepG2/ADR cells, while ATG5 upregulation partially reversed this effect (Figure 4D). Finally, the expression of autophagy-related proteins was notably decreased by overexpression of miR-155-5p, while ATG5 overexpression partially rescued the inhibitory effect of miR-155-5p mimics on autophagy in HepG2/ADR cells (Figure 4E). Altogether, these findings suggested that miR-155-5p sensitized HepG2/ ADR cells to ADR by targeting ATG5/BCL2.

\section{Discussion}

The major factor for chemotherapy failure is the chemoresistance of tumor cells [27]. Some drugs, such as 5-FU and verapamil, have been reported to act as reversal agents, but they have significant adverse effects that can largely reduce their clinical application $[28,29]$. Our study first verified the
A

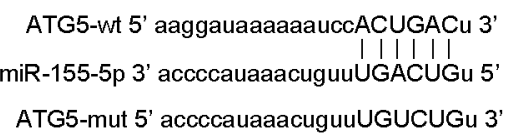

C

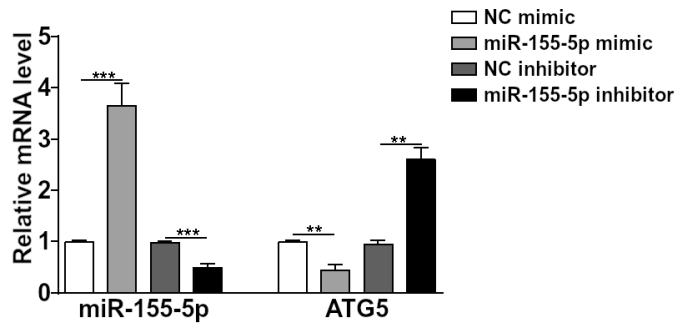

B

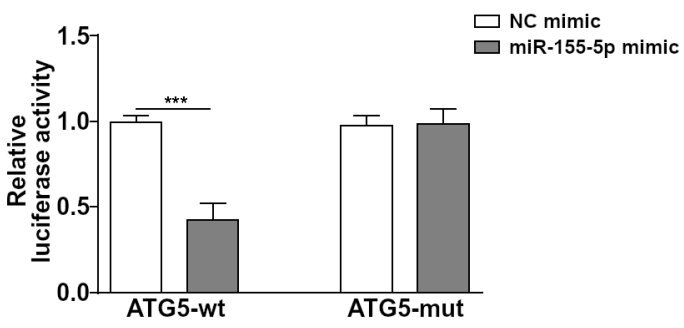

D
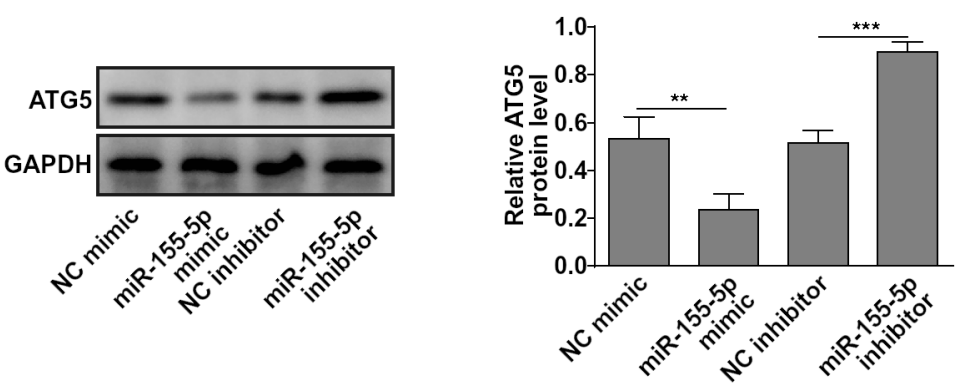

Figure 3. ATG5 was a direct binding target of miR-155-5p. A) The potential binding site between miR-155-5p and ATG5 was predicted by starBase v2.0 software. B) Luciferase activity was measured after co-transfection of miR-155-5p mimic and luciferase reporter plasmids carrying wild-type or mutant ATG5 3'-UTR in HepG2/ADR cells using the dual-luciferase reporter assay. The cells were treated with NC/miR-155-5p mimic or NC/miR-155$5 p$ inhibitor, and then the relative expression levels of miR-155-5p and ATG5 were detected by RT-qPCR (C) and western blot $(D) .{ }^{\star} p<0.05 ;{ }^{\star *} p<0.01$; ${ }^{* * *} \mathbf{p}<\mathbf{0 . 0 0 1}$ 
A

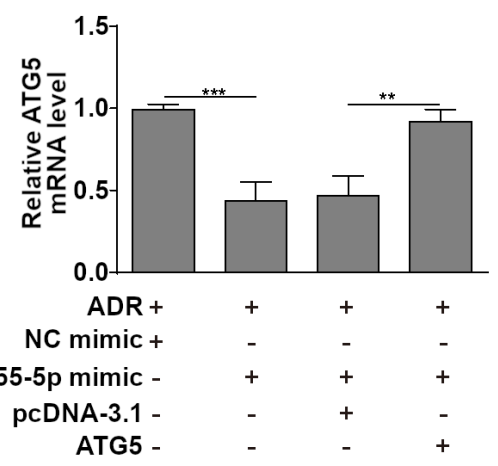

C

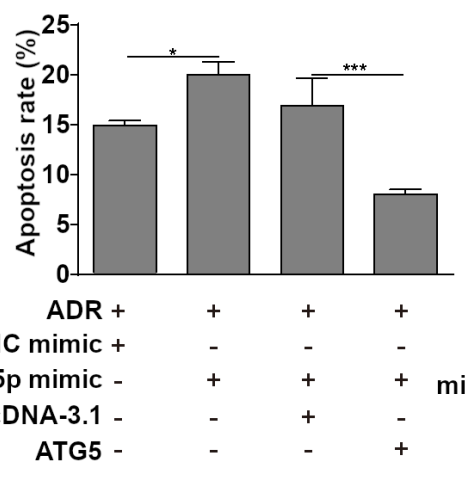

E

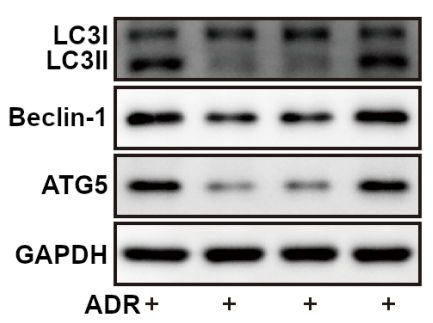

NC mimic +

miR-155-5p mimic -

pcDNA-3.1 -

F

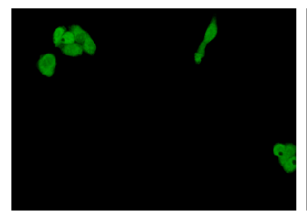

ADR+NC mimic

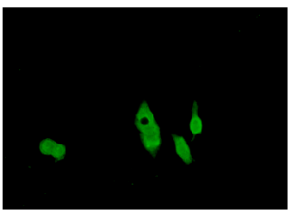

ADR
B

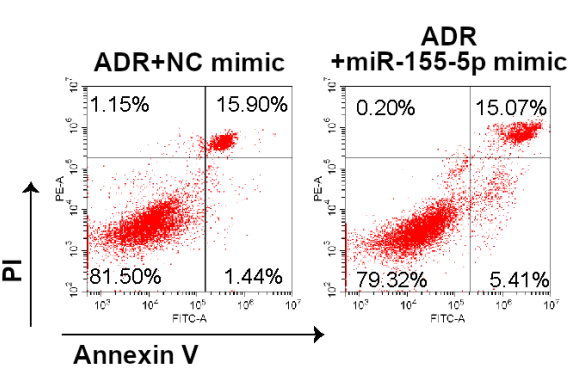

D

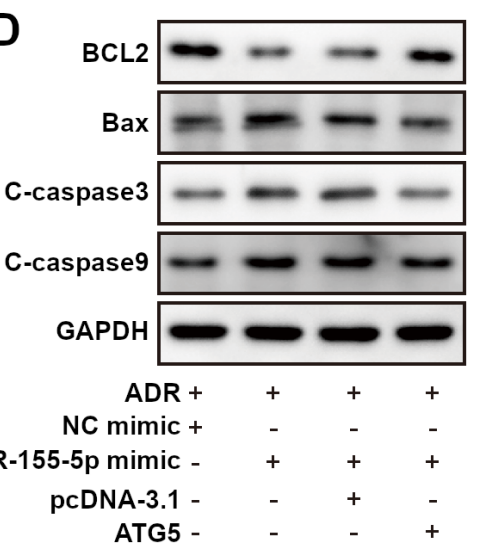

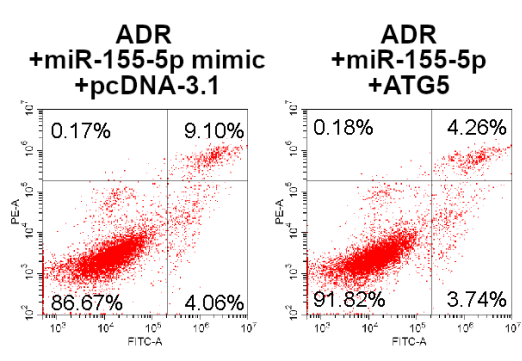

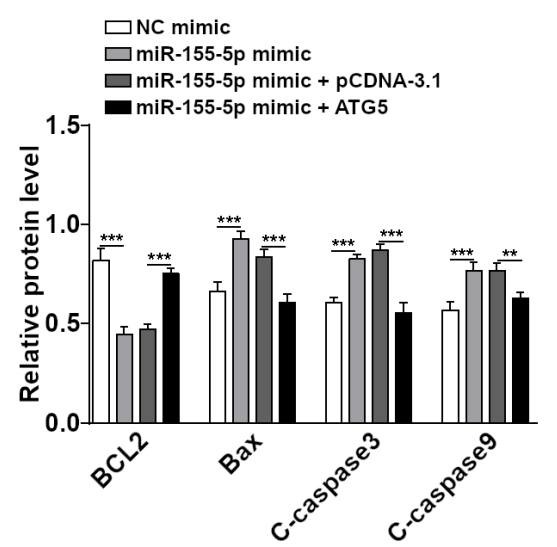

$\square$ NC mimic

$\square$ miR-155-5p mimic

$\square$ miR-155-5p mimic + pcDNA-3.1 - miR-155-5p mimic + ATG5

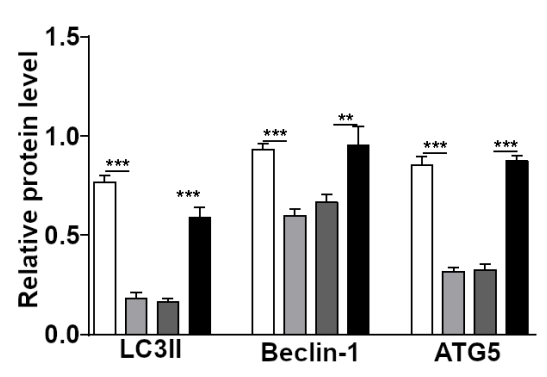

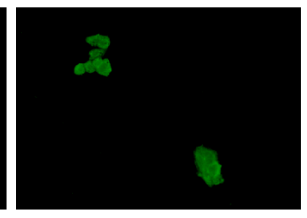

ADR +miR-155-5p mimic +pcDNA-3.1

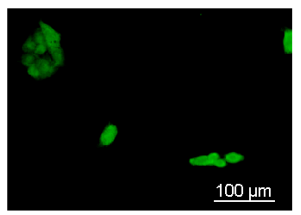

ADR

ADR
155-5p +ATG5

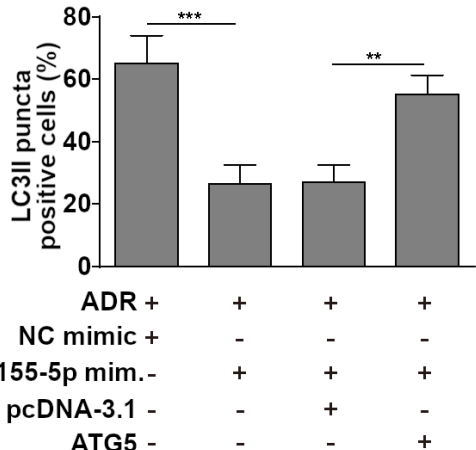

ATG5 -

Figure 4. miR-155-5p sensitized HepG2/ADR cells to ADR by targeting ATG5. A) HepG2/ADR cells were transfected with miR-155-5p mimic only or miR-155-5p mimic together with the ATG5 overexpression vector. Subsequently, the expression of ATG5 was detected by RT-qPCR. B) Cell apoptosis was measured by FACS. C) Quantitative analysis of (B). D) The expression of BCL2, Bax, cleaved caspase-3, and cleaved caspase-9 was assessed by western blot. E) The expression of LC3 was tested by immunofluorescence staining. F) LC3II, LC3I, ATG5, and Beclin-1 expression were evaluated by using western blot. ${ }^{\star} \mathbf{p}<0.05 ;{ }^{* *} \mathbf{p}<0.01 ;{ }^{* * *} \mathbf{p}<0.001$ 
inhibitory effect of miR-155-5p on ADR-resistant liver carcinoma in vitro. Recent studies have reported that miR-155-5p could act as a suppressor to reduce the chemoresistance of triple-negative breast cancer [30]. These results were similar to our present study, indicating that miR-155-5p can be regarded as a reversal agent for liver cancer treatment.

Our current results demonstrated that miR-155-5p overexpression significantly sensitized liver cancer cells to ADR treatment in vitro. Moreover, apoptosis in HepG2/ADR cells was significantly induced by the miR-155-5p mimic. In addition, our findings also suggested that miR-155-5p overexpression could notably activate pro-apoptotic proteins and inactivate anti-apoptotic proteins. Bax is considered an apoptosis-related and inflammation-related protein [31,32]. The upregulation of cleaved caspase- 3 could clearly induce apoptosis in cancer cells [33]. Cleaved caspase-9 is also involved in the apoptosis of tumor cells $[34,35]$. These studies were similar to our present study, indicating that miR-155-5p overexpression could induce apoptosis in resistant cancers in vitro via the regulation of apoptosis-related proteins.

Additionally, in our research, we found that miR-155-5p overexpression could inhibit autophagy in HepG2/ADR cells. Autophagy has been proven to be activated by capturing and degrading related proteins in lysosomes, which could result in recycling some molecules to maintain survival [36]. Autophagy also plays a regulatory role in mediating proteins and sustaining protein quality [37]. Autophagy dysfunction has been associated with multiple diseases [38]. In malignant tumors, autophagy can incur tumor chemoresistance in different contexts [39]. Kumar et al. indicated that autophagy was involved in drug resistance in malignant tumors [40]. Moreover, Meng et al. found that EGCG could reduce the resistance by downregulating autophagy and inhibiting cell death by targeting the phosphorylation of ERK in lung cancer [41]. Our findings were similar to the results of these studies, indicating that miR-155-5p sensitized HepG2/ ADR cells to ADR by inhibiting autophagy. Nevertheless, miR-155 has been reported to affect osteosarcoma MG-63 cell autophagy induced by adriamycin through regulation of the PTEN-PI3K/AKT/mTOR signaling pathway [42]. Our findings revealed that ATG5 and BCL2 were direct targets of miR-155. This difference may be due to different tumor types. Moreover, LC3 is an important regulator of yeast Atg8 [43]. A lipidated form of LC3, LC3II, is considered to be an important molecular marker in mammals and has been used to investigate the regulatory role of autophagy in neurodegenerative and neuromuscular diseases, tumorigenesis, and bacterial and viral infections [44]. Similarly, Beclin-1 has been proven to be involved in autophagy during tumorigenesis [45]. Sun et al. indicated that osteopontin could relieve early brain damage by modulating the association of autophagy and apoptosis after subarachnoid hemorrhage in vivo [45]. Similar to this study, our current research found that overexpression of miR-155-5p downregulated autophagy by decreasing the expression of Beclin-1 and LC3II in vitro.
It has been confirmed that miRNAs exert their biological functions through their target genes [46, 47]. In this study, a luciferase reporter assay indicated that ATG5 was a target gene of miR-155-5p in liver cancer. ATG5 is an autophagyrelated protein first regarded as a tumor promoter [48]. In addition, ATG5 could promote proliferation and metastasis, and inhibit breast cancer cell apoptosis [49]. A previous study found that rhein inhibited the autophagy of human cancer cells via downregulation of ATG5 [50]. Additionally, Ra et al. indicated that TRIM31 could promote Atg5/Atg7-independent autophagy in intestinal cells [51]. In addition, Lauzier et al. showed that ATG5 overexpression could promote the development of cancer in vitro [52]. In our research, the miR-155-5p mimic significantly decreased the expression of ATG5. However, ATG5 overexpression partially rescued the inhibitory effect of miR-155-5p on autophagy in liver cancer cells. Our findings were consistent with those of previous studies [53, 54]. Collectively, our results suggested that miR-155-5p exhibited an inhibitory effect on liver cancer cells by decreasing the level of ATG5.

In summary, our research first demonstrated that miR-155-5p sensitized HepG2/ADR cells to ADR by targeting ATG5, which may serve as a potential target for liver cancer treatment.

Supplementary information is available in the online version of the paper.

Acknowledgments: This work is supported by Hunan Science and Technology Department Project (No. 2017SK2144).

\section{References}

[1] LI HM, YE ZH. Microenvironment of liver regeneration in liver cancer. Chin J Integr Med 2017; 23: 555-560. https:// doi.org/10.1007/s11655-017-2806-0

[2] GRAVITZ L. Liver cancer. Nature 2014; 516: S1. https://doi. org/10.1038/516S1a

[3] LEI X, LEI Y, LI JK, DU WX, LI RG et al. Immune cells within the tumor microenvironment: biological functions and roles in cancer immunotherapy. Cancer Lett 2020; 470: 126-133. https://doi.org/10.1016/j.canlet.2019.11.009

[4] ZOU RC, LIANG Y, LI LL, TANG JZ, YANG YP et al. Bioinformatics Analysis Identifies Protein Tyrosine Kinase 7 (PTK7) as a Potential Prognostic and Therapeutic Biomarker in Stages I to IV Hepatocellular Carcinoma. Med Sci Monit 2019; 25: 8618-8627. https://doi.org/10.12659/MSM.917142

[5] XIA X, WANG Q, YE T, LIU Y, LIU D et al. NRF2/ABCB1mediated efflux and PARP1-mediated dampening of DNA damage contribute to doxorubicin resistance in chronic hypoxic HepG2 cells. Fundam Clin Pharmacol 2020; 34: 41-50. https://doi.org/10.1111/fcp.12505

[6] MENG F, ZHANG S, SONG R, LIU Y, WANG J et al. NCAPG2 overexpression promotes hepatocellular carcinoma proliferation and metastasis through activating the STAT3 and NF-kappaB/miR-188-3p pathways. EBioMedicine 2019; 44: 237-249. https://doi.org/10.1016/j.ebiom.2019.05.053 
[7] WEI D, YU G, ZHAO Y. MicroRNA-30a-3p inhibits the progression of lung cancer via the PI3K/AKT by targeting DNA methyltransferase 3a. Onco Targets Ther 2019; 12: 7015-7024. https://doi.org/10.2147/OTT.S213583

[8] QIAN K, LI Q, DENG W, XIANG X. Multiple-Scales Integrative Analysis of MicroRNAs Unveils Biomarkers and Key Regulatory Connections for Hepatocellular Carcinoma. Crit Rev Eukaryot Gene Expr 2019; 29: 189-241. https://doi. org/10.1615/CritRevEukaryotGeneExpr.2019025931

[9] CHEN Q, CHEN Z, CAO S, GUO B, CHEN Y et al. Role of CircRNAs_100395 in Proliferation and Metastases of Liver Cancer. Med Sci Monit 2019; 25: 6181-6192. https://doi. org/10.12659/MSM.915963

[10] SUN SN, HU S, SHANG YP, LI LY, ZHOU H et al. Relevance function of microRNA-708 in the pathogenesis of cancer. Cell Signal 2019; 63: 109390. https://doi.org/10.1016/j.cellsig.2019.109390

[11] YAO J, CHENG Y, ZHANG D, FAN J, ZHAO Z et al. Identification of key genes, MicroRNAs and potentially regulated pathways in alcoholic hepatitis by integrative analysis. Gene 2019; 720: 144035. https://doi.org/10.1016/j. gene.2019.144035

[12] LIVINGSTONE MC, JOHNSON NM, ROEBUCK BD, KENSLER TW, GROOPMAN JD. Serum miR-182 is a predictive biomarker for dichotomization of risk of hepatocellular carcinoma in rats. Mol Carcinog 2019; 58: 2017-2025. https://doi.org/10.1002/mc.23093

[13] MA S, SUN J, GUO Y, ZHANG P, LIU Y et al. Combination of AAV-TRAIL with miR-221-Zip Therapeutic Strategy Overcomes the Resistance to TRAIL Induced Apoptosis in Liver Cancer. Theranostics 2017; 7: 3228-3242. https://doi. org/10.7150/thno.19893

[14] CHAI Z, YIN X, CHEN J, SHI J, SUN J et al. MicroRNA-101 modulates cisplatin chemoresistance in liver cancer cells via the DNA-PKcs signaling pathway. Oncol Lett 2019; 18: 3655-3663. https://doi.org/10.3892/ol.2019.10674

[15] WANG T, LI B, WANG Z, YUAN X, CHEN C et al. miR155-5p Promotes Dorsal Root Ganglion Neuron Axonal Growth in an Inhibitory Microenvironment via the cAMP/ PKA Pathway. Int J Biol Sci 2019; 15: 1557-1570. https://doi. org/10.7150/ijbs.31904

[16] ZHAO Y, RAN Z, JIANG Q, HU N, YU B et al. Vitamin D Alleviates Rotavirus Infection through a Microrna-155-5p Mediated Regulation of the TBK1/IRF3 Signaling Pathway In Vivo and In Vitro. Int J Mol Sci 2019; 20: 3562. https://doi. org/10.3390/ijms20143562

[17] TONG L, AO Y, ZHANG H, WANG K, WANG Y et al. Long noncoding RNA NORAD is upregulated in epithelial ovarian cancer and its downregulation suppressed cancer cell functions by competing with miR-155-5p. Cancer Med 2019; 8: 4782-4791. https://doi.org/10.1002/cam4.2350

[18] ZHANG L, CHEN T, YAN L, XU H, WANG Y et al. MiR155-3p acts as a tumor suppressor and reverses paclitaxel resistance via negative regulation of MYD88 in human breast cancer. Gene 2019; 700: 85-95. https://doi.org/10.1016/j. gene.2019.02.066

[19] LI YJ, LEI YH, YAO N, WANG CR, HU N et al. Autophagy and multidrug resistance in cancer. Chin J Cancer 2017; 36: 52. https://doi.org/10.1186/s40880-017-0219-2
[20] KLIONSKY DJ. Look people, "Atg" is an abbreviation for "autophagy-related." That's it. Autophagy 2012; 8: 12811282. https://doi.org/10.4161/auto.21812

[21] ROMANOV J, WALCZAK M, IBIRICU I, SCHUCHNER $\mathrm{S}$, OGRIS $\mathrm{E}$ et al. Mechanism and functions of membrane binding by the Atg5-Atg12/Atg16 complex during autophagosome formation. EMBO J 2012; 31: 4304-4317. https:// doi.org/10.1038/emboj.2012.278

[22] AKIND, WANGSK, HABIBZADEGAH-TARIP,LAWB, OSTROV D et al. A novel ATG4B antagonist inhibits autophagy and has a negative impact on osteosarcoma tumors. Autophagy 2014; 10: 2021-2035. https://doi.org/10.4161/auto.32229

[23] LEFORT S, JOFFRE C, KIEFFER Y, GIVEL AM, BOURACHOT B et al. Inhibition of autophagy as a new means of improving chemotherapy efficiency in high-LC3B triple-negative breast cancers. Autophagy 2014; 10: 2122 2142. https://doi.org/10.4161/15548627.2014.981788

[24] SUI X, CHEN R, WANG Z, HUANG Z, KONG $\mathrm{N}$ et al. Autophagy and chemotherapy resistance: a promising therapeutic target for cancer treatment. Cell Death Dis 2013; 4: e838. https://doi.org/10.1038/cddis.2013.350

[25] HE Y, ZHU Q, CHEN M, HUANG Q, WANG W et al. The changing $50 \%$ inhibitory concentration (IC50) of cisplatin: a pilot study on the artifacts of the MTT assay and the precise measurement of density-dependent chemoresistance in ovarian cancer. Oncotarget 2016; 7: 70803-70821. https:// doi.org/10.18632/oncotarget.12223

[26] LIU H, LEI C, HE Q, PAN Z, XIAO D et al. Nuclear functions of mammalian MicroRNAs in gene regulation, immunity and cancer. Mol Cancer 2018; 17: 64. https://doi. org/10.1186/s12943-018-0765-5

[27] WU Q, YANG Z, NIE Y, SHI Y, FAN D. Multi-drug resistance in cancer chemotherapeutics: mechanisms and lab approaches. Cancer Lett 2014; 347: 159-166. https://doi. org/10.1016/j.canlet.2014.03.013

[28] GU J, LI Z, ZHOU J, SUN Z, BAI C. Response prediction to oxaliplatin plus 5-fluorouracil chemotherapy in patients with colorectal cancer using a four-protein immunohistochemical model. Oncol Lett 2019; 18: 2091-2101. https://doi. org/10.3892/ol.2019.10474

[29] XU J, TASNEEN R, PELOQUIN CA, ALMEIDA DV, LI SY et al. Verapamil Increases the Bioavailability and Efficacy of Bedaquiline but Not Clofazimine in a Murine Model of Tuberculosis. Antimicrob Agents Chemother 2017; 62: e01692-17. https://doi.org/10.1128/AAC.01692-17

[30] OUYANG M, LI Y, YE S, MA J, LU L et al. MicroRNA profiling implies new markers of chemoresistance of triple-negative breast cancer. PLoS One 2014; 9: e96228. https://doi. org/10.1371/journal.pone.0096228

[31] CAGLAYAN C, KANDEMIR FM, DARENDELIOGLU E, YILDIRIM S, KUCUKLER S et al. Rutin ameliorates mercuric chloride-induced hepatotoxicity in rats via interfering with oxidative stress, inflammation and apoptosis. J Trace Elem Med Biol 2019; 56: 60-68. https://doi.org/10.1016/j. jtemb.2019.07.011

[32] WANG S, CHI Q, HU X, CONG Y, LI S. Hydrogen sulfideinduced oxidative stress leads to excessive mitochondrial fission to activate apoptosis in broiler myocardia. Ecotoxicol Environ Saf 2019; 183: 109578. https://doi.org/10.1016/j. ecoenv.2019.109578 
[33] CROWLEY LC, WATERHOUSE NJ. Detecting Cleaved Caspase-3 in Apoptotic Cells by Flow Cytometry. Cold Spring Harb Protoc 2016; 2016. https://doi.org/10.1101/pdb. prot087288

[34] KUIDA K. Caspase-9. Int J Biochem Cell Biol 2000; 32: 121124. https://doi.org/10.1016/s1357-2725(99)00024-2

[35] LI Y, ZHOU M, HU Q, BAI XC, HUANG W et al. Mechanistic insights into caspase- 9 activation by the structure of the apoptosome holoenzyme. Proc Natl Acad Sci U S A 2017; 114: 1542-1547. https://doi.org/10.1073/pnas.1620626114

[36] AMARAVADI R, KIMMELMAN AC, WHITE E. Recent insights into the function of autophagy in cancer. Genes Dev 2016; 30: 1913-1930. https://doi.org/10.1101/ gad.287524.116

[37] HUO Y, CAI H, TEPLOVA I, BOWMAN-COLIN C, CHEN $\mathrm{G}$ et al. Autophagy opposes p53-mediated tumor barrier to facilitate tumorigenesis in a model of PALB2-associated hereditary breast cancer. Cancer Discov 2013; 3: 894-907. https://doi.org/10.1158/2159-8290.CD-13-0011

[38] CHOURASIA AH, TRACY K, FRANKENBERGER C, BOLAND ML, SHARIFI MN et al. Mitophagy defects arising from BNip3 loss promote mammary tumor progression to metastasis. EMBO Rep 2015; 16: 1145-1163. https://doi. org/10.15252/embr.201540759

[39] CICCHINI M, CHAKRABARTI R, KONGARA S, PRICE $\mathrm{S}$, NAHAR R et al. Autophagy regulator BECN1 suppresses mammary tumorigenesis driven by WNT1 activation and following parity. Autophagy 2014; 10: 2036-2052. https:// doi.org/10.4161/auto.34398

[40] KUMAR A, SINGH UK, CHAUDHARY A. Targeting autophagy to overcome drug resistance in cancer therapy. Future Med Chem 2015; 7: 1535-1542. https://doi.org/10.4155/ fmc. 15.88

[41] MENG J, CHANG C, CHEN Y, BI F, JI C et al. EGCG overcomes gefitinib resistance by inhibiting autophagy and augmenting cell death through targeting ERK phosphorylation in NSCLC. Onco Targets Ther 2019; 12: 6033-6043. https:// doi.org/10.2147/OTT.S209441

[42] WANG L, TANG B, HAN H, MAO D, CHEN J et al. miR155 Affects Osteosarcoma MG-63 Cell Autophagy Induced by Adriamycin Through Regulating PTEN-PI3K/AKT/ mTOR Signaling Pathway. Cancer Biother Radiopharm 2018; 33: 32-38. https://doi.org/10.1089/cbr.2017.2306

[43] TANIDA I, UENO T, KOMINAMI E. LC3 conjugation system in mammalian autophagy. Int J Biochem Cell Biol 2004; 36: 2503-2518. https://doi.org/10.1016/j.biocel.2004.05.009

[44] TANIDA I, UENO T, KOMINAMI E. LC3 and Autophagy. Methods Mol Biol 2008; 445: 77-88. https://doi. org/10.1007/978-1-59745-157-4_4
[45] SUN CM, ENKHJARGAL B, REIS C, ZHOU KR, XIE ZY et al. Osteopontin attenuates early brain injury through regulating autophagy-apoptosis interaction after subarachnoid hemorrhage in rats. CNS Neurosci Ther 2019; 25: 11621172. https://doi.org/10.1111/cns.13199

[46] BACKES C, MEESE E, KELLER A. Specific miRNA Disease Biomarkers in Blood, Serum and Plasma: Challenges and Prospects. Mol Diagn Ther 2016; 20: 509-518. https://doi. org/10.1007/s40291-016-0221-4

[47] BERNARDO BC, OOI JY, LIN RC, MCMULLEN JR. miRNA therapeutics: a new class of drugs with potential therapeutic applications in the heart. Future Med Chem 2015; 7: 1771-1792. https://doi.org/10.4155/fmc.15.107

[48] LECHAUVE C, KEITH J, KHANDROS E, FOWLER S, MAYBERRY $\mathrm{K}$ et al. The autophagy-activating kinase ULK1 mediates clearance of free alpha-globin in beta-thalassemia. Sci Transl Med 2019; 11: eaav4881. https://doi.org/10.1126/ scitranslmed.aav4881

[49] HAN Q, DENG Y, CHEN S, CHEN R, YANG M et al. Downregulation of ATG5-dependent macroautophagy by chaperone-mediated autophagy promotes breast cancer cell metastasis. Sci Rep 2017; 7: 4759. https://doi.org/10.1038/ s41598-017-04994-X

[50] LI Y, SHEN F, BAO Y, CHEN D, LU H. Apoptotic effects of rhein through the mitochondrial pathways, two death receptor pathways, and reducing autophagy in human liver L02 cells. Environ Toxicol 2019; 34: 1292-1302. https://doi. org/10.1002/tox.22830

[51] RA EA, LEE TA, WON KIM S, PARK A, CHOI HJ et al. TRIM31 promotes Atg5/Atg7-independent autophagy in intestinal cells. Nat Commun 2016; 7: 11726. https://doi. org/10.1038/ncomms 11726

[52] LAUZIER A, NORMANDEAU-GUIMOND J, VAILLANCOURT-LAVIGUEUR V, BOIVIN V, CHARBONNEAU $\mathrm{M}$ et al. Colorectal cancer cells respond differentially to autophagy inhibition in vivo. Sci Rep 2019; 9: 11316. https:// doi.org/10.1038/s41598-019-47659-7

[53] JIAO L, ZHANG HL, LI DD, YANG KL, TANG J et al. Regulation of glycolytic metabolism by autophagy in liver cancer involves selective autophagic degradation of HK2 (hexokinase 2). Autophagy 2018; 14: 671-684. https://doi.org/10.10 80/15548627.2017.1381804

[54] ORDONEZ R, FERNANDEZ A, PRIETO-DOMINGUEZ $\mathrm{N}$, MARTINEZ L, GARCIA-RUIZ C et al. Ceramide metabolism regulates autophagy and apoptotic cell death induced by melatonin in liver cancer cells. J Pineal Res 2015; 59: 178-189. https://doi.org/10.1111/jpi.12249 